\title{
Mycobacterium chelonei: friend or foe?
}

\section{To the Editors:}

In the paper by DAILLOUx et al. [1], the authors reminded us that in order to establish the diagnosis of nontuberculous mycobacterial (NTM) infection, it is necessary to combine data obtained from the mycobacterial laboratory with the clinician's assessments. Furthermore, it was also stated that the adherence to published guidelines should improve the diagnosis of these diseases. We completely agree with the authors and would like to emphasise the importance of using these guidelines even when all the results seem to point to a straightforward diagnosis.

In our clinic, a patient presented with dyspnoea, nonproductive cough and infiltrate on radiography. Computed tomography of the thorax revealed a diffuse pulmonary infiltrate in the right upper lobe. The patient underwent a bronchoscopy, which was unremarkable, and bronchoalveolar lavage, which revealed Mycobacterium chelonei in culture. Given the clinical picture, all evidence pointed towards a diagnosis of pulmonary M. cheloni. We had planned to start treatment and therefore consulted the guidelines on treating this organism. American Thoracic Society (ATS) guidelines on the diagnosis and treatment of NTM infection were reviewed. We could see that the diagnosis requires three positive bronchial washing cultures (or two positive cultures and one positive acid-fast bacilli (AFB) smear) in association with characteristic radiographical findings [2]. If only one bronchial wash is available, the diagnosis can be made with a $2+, 3+$ or $4+$ AFB smear or $2+$, $3+$ or $4+$ growth on solid media. Otherwise, a single positive result may represent a contaminant or persistent colonisation [2]. We arranged for the patient to return to our clinic and have some follow-up films and a repeat bronchoscopy. We were also concerned that this patient may have even have been immunocompromised, and planned a strategy to evaluate this problem. A number of days before the repeat bronchoscopy and imaging were due, the microbiology laboratory informed us that three other patients from the bronchoscopy list were culture positive for $M$. chelonei. Given the coincidental finding of this unusual organism in four patients from one bronchoscopy list, the possibility of contamination was raised. Further assessment and investigation by the laboratory duly found and proved the organism to be a contaminant. Over the following few weeks, the patient's cough became productive (with sputum culture yielding bacterial pathogens) and the findings completely resolved over time.

M. chelonei is a nontuberculous mycobacterium that is widely distributed throughout the world. It has been found in water and sewage, and rapidly grows in culture and can cause infections of the lungs, skin and lymph nodes. Disseminated disease has also been described, but this is almost exclusively seen in the immunocompromised. Infection can lead to bronchiectasis and death may result from dissemination of extensive pulmonary disease.

Its incidence and prevalence are increasing around the world for this very reason and, therefore, more difficulties will arise in the future in identifying this organism as a commensal or pathogen. In the paper by HENRY et al. [3], the authors revealed that NTM increased between 1995-1999 in a non-HIV population and that $74 \%$ of the diagnosed patients had pulmonary disease. They also demonstrated a better outcome in patients who received treatment according to the ATS [2] and British Thoracic Society [4] guidelines, as compared with treatments given prior to the introduction of these guidelines.

This case highlights the need to follow the guidelines mentioned by DAILloux et al. [1], so that we are sure of the diagnosis no matter how suspicious we are from a clinical and diagnostic perspective before we start prematurely treating Mycobacterium chelonei, "the friend", with potentially toxic drugs, rather than carefully assessing and properly treating Mycobacterium chelonei, "the foe"!

\section{E.E. McGrath and N. Qureshi}

Dept of Respiratory Medicine, Rotherham District General Hospital, Rotherham, UK.

\section{STATEMENT OF INTEREST}

None declared.

\section{REFERENCES}

1 Dailloux M, Abalain ML, Laurain C, et al. Respiratory infections associated with nontuberculous mycobacteria in non-HIV patients. Eur Respir J 2006; 28: 1211-1215.

2 Diagnosis and treatment of disease caused by nontuberculous mycobacteria. This official statement of the American Thoracic Society was approved by the Board of Directors, March 1997. Medical Section of the American Lung Association. Am J Respir Crit Care Med 1997; 156: S1-S25.

3 Henry MT, Inamdar L, O'Riordain D, Schweiger M, Watson JP. Nontuberculous mycobacteria in non-HIV patients: epidemiology, treatment and response. Eur Respir J 2004; 23: 741-746.

4 Management of opportunist mycobacterial infections: Joint Tuberculosis Committee Guidelines 1999. Subcommittee of the Joint Tuberculosis Committee of the British Thoracic Society. Thorax 2000; 55: 210-218. 